\title{
CCN2 reduction mediates protective effects of BMP7 treatment in obstructive nephropathy
}

\author{
Lucas L. Falke $^{1}$ - Jan Willem Leeuwis ${ }^{1}$ Karen M. Lyons ${ }^{2} \cdot$ Christine L. Mummery $^{3}$. \\ Tri Q. Nguyen ${ }^{1} \cdot$ Roel Goldschmeding ${ }^{1}$
}

Received: 27 June 2016 / Accepted: 6 October 2016 / Published online: 20 October 2016

(C) The Author(s) 2016. This article is published with open access at Springerlink.com

\begin{abstract}
Treatment with rhBMP7 exerts profound protective effects in a wide variety of experimental models of renal disease. However, little is known about how these protective effects are mediated, and which cells in the kidney are targeted by exogenous rhBMP7 treatment. To determine if rhBMP7 increases glomerular and tubulointerstitial canonical BMP signaling, we performed Unilateral Ureteral Obstruction (UUO, a widely used obstructive nephropathy model) in mice reporting transcriptional activity downstream of canonical BMP signaling by the expression of GFP under the BMP Responsive Element of the Id1 promoter (BRE:gfp mice). We also analysed the impact of rhBMP7 treatment on severity of the UUO phenotype, on TGF $\beta$ signaling, and on expression of CCN2 (CTGF). Despite profound protective effects with respect to morphological damage, macrophage infiltration, and fibrosis, no significant difference in GFPexpression was observed upon rhBMP7 administration. Also TGF $\beta$ signalling was similar in rhBMP7 and vehicle treated mice, but $\mathrm{CCN} 2$ expression in obstructed kidneys was significantly reduced by rhBMP7 treatment. Of
\end{abstract}

Electronic supplementary material The online version of this article (doi:10.1007/s12079-016-0358-2) contains supplementary material, which is available to authorized users.

Lucas L. Falke

lucasfalke@hotmail.com

1 Department of Pathology, Kidney Group, University Medical Centre Utrecht, H04.312, Heidelberglaan 100, 3584, CX

Utrecht, The Netherlands

2 Department of Molecular, Cell and Developmental Biology, University of California, Los Angeles, CA, USA

3 Department of Anatomy and Embryology, Leiden University Medical Centre, Leiden, The Netherlands note, in heterozygous $\mathrm{CCN} 2$ mice $(\mathrm{CCN} 2+--)$ treatment with rhBMP7 did not (further) reduce the severity of kidney damage in the UUO-model. These data suggest that protection against obstructive nephropathy by exogenous rhBMP7 treatment relies primarily on non-canonical BMP signaling, and may be mediated in large part by downregulation of CCN2 expression.

Keywords BMP7 · TGF $\beta$ - CCN2 - CKD · UUO · Kidney

$\begin{array}{ll}\text { Abbreviations } \\ \text { BMP7 } & \text { Bone Morphogenetic Protein } 7 \\ \text { CCN2 } & \text { Cyr61-CTGF-Nov family protein 2 } \\ \text { CTGF } & \text { Connective Tissue Growth Factor } \\ \text { CKD } & \text { Chronic Kidney Disease } \\ \text { UUO } & \text { Unilateral Ureteral Obstruction } \\ \text { BW } & \text { Body Weight } \\ \text { OBK } & \text { Obstructed Kidney } \\ \text { CLK } & \text { Contralateral Kidney } \\ \text { GFP } & \text { Green Fluorescent Protein } \\ \text { TGF } \beta & \text { Transforming Growth Factor } \beta \\ \text { LTA } & \text { Lotus tetragonolobus Agglutinin }\end{array}$

\section{Introduction}

Irrespective of underlying aetiology, chronic kidney disease (CKD) involves structural changes, and ultimately loss of function and fibrosis. Although, there is no effective treatment, several potential targets for intervention in CKD progression have been identified. Transforming Growth Factor beta $(\mathrm{TGF} \beta)$ is generally regarded as the main culprit driving CKD progression (Meng et al. 2015). Numerous studies targeting TGF $\beta$ in various experimental diseases have yielded 
favourable results, but recent clinical trials have questioned efficacy of available interventions in human CKD (Akhurst and Hata 2012; Muñoz-Félix et al. 2015) (Clinicaltrial.gov numbers NCT00464321 and NCT01113801).

The administration of recombinant human BMP7 (rhBMP7; Bone Morphogenetic Protein 7) has been proposed as an attractive alternative intervention to stop progression of CKD. Several landmark papers have shown efficacy of BMP7 treatment in a wide range of experimental models of renal disease including diabetic nephropathy, obstructive uropathy, nephron loss and ischemic injury (Vukicevic et al. 1998; Hruska et al. 2000; Morrissey et al. 2002; Wang et al. 2003; Zeisberg et al. 2003; Dube et al. 2004; Sugimoto et al. 2007), and a BMP-mimetic (THR-185) is under study in a phase II clinical trial (Clinicaltrial.gov number NCT01830920). BMP7 treatment is considered to attenuate experimental CKD at least in part by antagonizing TGF $\beta$ (Wang and Hirschberg 2003; Zeisberg et al. 2003). BMP7 is required for kidney development and remains highly expressed during adult life (Dudley et al. 1995). Although also several other BMPs are expressed in the kidney throughout development and adulthood, including BMP4 and BMP6, the potential therapeutic effects of BMP7 are considered most potent (Zeisberg et al. 2003; Dendooven et al. 2011).

Despite all evidence supporting BMP7 efficacy in CKD, the identity and localization of cells responding to exogenous BMP7 treatment remain to be identified. Previous studies in BMP canonical signaling reporter mice (BRE:gfp mice) identified glomerular and collecting duct cells to have high endogenous BMP signalling activity (Leeuwis et al. 2011). Signaling activity in the glomeruli and medulla dropped upon Unilateral Ureteral Obstruction (UUO), but it increased in the proximal tubular compartment. However, if and to what extent exogenous BMP7 therapeutic efficacy might involve restoration of canonical signaling activity in these particular cells or other nephron segments or cell types is still unclear.

CCN2, also known as Connective Tissue Growth Factor (CTGF) is yet another factor involved in CKD progression (Falke et al. 2014). CCN2 contributes to fibrosis by modulating signaling activity in BMP7, TGF $\beta$, and other signaling pathways (Abreu et al. 2002; Nguyen et al. 2008). CCN2 expression is increased in essentially all progressive kidney diseases, and CCN2 inhibition decreases loss of function and fibrosis (Ito et al. 1998; Falke et al. 2014; Ren et al. 2015). CCN2 can bind to BMP7 thereby inhibiting canonical SMAD1/5/8 signalling, and as such might also be an important determinant of the efficacy of BMP7 treatment (Nguyen et al. 2008).

In this study, we set out to shed more light on the mode of action of exogenous BMP7 therapy by analysing distribution of transcriptional activity downstream of canonical BMP signaling, and the associated complex interplay between BMP7, TGF $\beta$, and CCN2 in rhBMP7 treated BRE-GFP reporter mice subjected to UUO.

\section{Materials and methods}

\section{Animals}

Generation of bone morphogenetic protein responsive element (BRE); Green Fluorescent Protein (GFP) reporter mice has been described in detail elsewhere (Monteiro et al. 2008). Briefly, these mice express GFP under control of the BRE in the Id1 gene promoter, thus reporting transcriptional activity downstream canonical BMP signaling. Male BRE:gfp mice on a $\mathrm{C} 57 \mathrm{Bl6} / \mathrm{J}$ background were used for this study. CCN2 hemizygous $\mathrm{KO}$ mice were used to estimate the relative contribution of CCN2 reduction to the therapeutic effect of exogenous BMP7. CCN2 (hemizygous) KO mice have been described previously (Ivkovic et al. 2003). Mice were kept on a 12-h light/day cycle with food and water ad libitum. All work was carried out with approval of the Experimental Animal Ethics Committee of the University of Utrecht.

\section{Unilateral ureteral obstruction/BMP7 administration}

Mice were subjected to Unilateral Ureteral Obstruction under general isoflurane anaesthesia. The left flank was incised and the ureter was exposed and tied off using silk sutures. Directly after ligation, a depot of $300 \mu \mathrm{g} / \mathrm{kg}$ rhBMP7 (dissolved in PBS; kindly provided by Stryker, Kalamazoo, MI) per mouse was left intraperitoneally (i.p.). The wound was closed using 5-0 vicryl sutures (Ethicon, Sommerville, NJ). Mice received an additional $300 \mu \mathrm{g} / \mathrm{kg}$ rhBMP7 i.p. on day 2, 4 and $6(n=9)$. Vehicle (PBS) injected mice were used as control $(n=6) .3$ mice were used for CCN2 hemizygous groups. At day 7 mice were killed by ketamin, xylazine and acepromazine overdose. Kidney tissue was fixed in fresh $4 \%$ paraformaldehyde solution and embedded in paraffin, or snap frozen and stored at $-80{ }^{\circ} \mathrm{C}$ until further processing.

\section{(Immuno)histochemistry}

Sections $(3 \mu \mathrm{m})$ were cut from paraffin blocks, deparaffinised and rehydrated. For assessment of morphological changes, sections were stained with Periodic Acid Schiff using standard methods. 10 random cortical fields of PAS stained sections were scored on a five-point scale for tubular atrophy or dilatation $(0=0-10 \%, 1=10-25 \%, 2=25-50 \%, 3=50-75 \%$, $4=75-100 \%$ ). Masson Tri Chrome (MTC) and Sirius Red staining was performed using standardized protocols at the clinical diagnostics laboratory of our department.

Direct fluorescence of 20 glomeruli per kidney was assessed in images acquired by confocal laser scanning microscopy (CLSM), followed by ImageJ analysis of signal intensity. Likewise, direct tubuloinsterstitial GFP signal was assessed in CLSM images by analyzing area positivity and 
single channel pixel intensity of 10 cortical fields from which glomeruli were deselected.

For immunohistochemistry, slides were boiled in EDTA or citrate buffer for antigen retrieval where appropriate, and incubated with the following antibodies: anti-GFP (Ab290 , rabbit polyclonal, Citrate, 1:1000, Abcam, Cambridge, UK), HRP-linked LTA (Citrate, 1:32, Sigma-Aldrich, St. Louis, MO), anti-CCN2 (L20, goat polyclonal, Citrate, 1:200, Santa-Cruz Biotechnology, Santa Cruz, CA), anti-F4/80 (Fresh Frozen tissue, rat monoclonal, 1:3000, Serotec/Biorad antibodies, Oxford, UK) or anti- $\alpha$ SMA (Ab5694, rabbit polyclonal, EDTA, 1:200, Abcam). Ready to use Horse Radish Peroxidase (HRP) linked BrightVision (ImmunoLogic, Duiven, NL) species specific secondary antibody solution, followed by NovaRed (Vector laboratories, Burlingham, CA) or 3,3'-diaminobenzidine (Sigma Aldrich, St. Louis, $\mathrm{MO}$ ) were applied for chromogen color development to visualize bound primary antibody.

The number of proximal tubule cross sections per cortical surface area was counted in 10 random fields per kidney (200× magnified). Using Photoshop (Adobe, San Jose, CA), a colour selection of positively stained area was made after which pictures were dichotomized in IHC positive and staining negative pixels. Using this method, the percentage positive cross sectional surface area for GFP, F4/ 80 , and $\alpha$ SMA respectively, was determined in 10 cortical fields photographed at $200 \times$ magnification by ImageJ analysis $(\mathrm{NIH})$.

For glomerular GFP IHC area positivity, pictures of 20 glomeruli per kidney were converted into binary images. Supplemental Fig. 1 A shows one example of a binary image of the CLK from the vehicle group illustrating what is regarded as total and positive area for calculation of positive area percentage. The red dotted lines define the circumference of two glomeruli. The enclosed total glomerular crosssectional surface is used for calculation of glomerular positive area percentage. The total glomerular cross-sectional surface area is excluded for calculation of tubulointerstitial positive area percentage.

Table 1 Body and kidney weights of both treatment groups. Average (+/- standard deviation) is shown

\section{RT-qPCR}

Full Kidney cortex mRNA was isolated using Trizol and $3000 \mathrm{ng}$ of mRNA was reversely transcribed into cDNA. RTqPCR was performed on a LightCycler480 (Roche, Basel, Switzerland), using commercially available TaqMan primer assays (Thermo Fisher/Life technologies, Waltham, MA): (Yhwaz; Mm03950126_s1, Ctgf, Mm00515790_g1; Tgfß1, Mm01178820_m1; Col1 22 , Mm00483888_m1; Pai1, Mm00435860_m1). Sybr green GFP primers and probe were designed with Primer Express (Applied Biosystems, Foster City, CA) and purchased from Eurogentec (Maastricht, The Netherlands) (primers) and Applied Biosystems (probe). Yhwaz expression was used as internal reference. Relative expression values were calculated using the $\Delta \Delta \mathrm{CT}$ method.

\section{Statistics}

All statistical analyses were performed using Graphpad Prism (GraphPad, LaJolla, CA). ANOVA with Tukey post-hoc correction for multiple testing or Student T-test were used where appropriate. A $p$-value below 0.05 was considered statistically significant.

\section{Results}

\section{Exogenous BMP7 does not increase BRE:gfp reporter signal for transcriptional activity downstream of canonical BMP7 signaling}

Body weight, kidneys weights and kidney weight/body weight ratios of rhBMP7 treated BRE:gfp mice were not significantly different from those in vehicle treated mice (See Table 1). BRE:gfp mice express GFP under a SMAD binding element in the Id1 promotor region and thereby report transcriptional activity downstream of canonical BMP signaling (Korchynskyi and Dijke ten 2002; Monteiro et al. 2008). Glomerular GFP intensity tended to be slightly lower in

\begin{tabular}{llll}
\hline & Vehicle $(\mathrm{n}=6)$ & rhBMP7 $(\mathrm{n}=9)$ & $p$-value \\
\hline BW start (g) & $\mathbf{2 5 . 9 2 ( + / - 4 . 4 1 )}$ & $\mathbf{2 5 . 8 8}(+/-4.32)$ & 0.99 \\
BW end (g) & $\mathbf{2 5 . 8 7 ( + / - 3 . 8 2 )}$ & $\mathbf{2 4 . 8 4}(+/-3.52)$ & 0.61 \\
BW end/BW start & $\mathbf{1}(+/-0.05)$ & $\mathbf{0 . 9 6}(+/-0.04)$ & 0.15 \\
CLKW (mg) & $\mathbf{2 1 1 . 6}(+/-48.2)$ & $\mathbf{2 1 1}(+/-47.69)$ & 0.98 \\
OBKW (mg) & $\mathbf{1 9 4 . 6 7 ( + / - 3 7 . 3 3 )}$ & $\mathbf{1 9 9 . 8 8}(+/-25.98)$ & 0.76 \\
CLKW (mg/g BWs) & $\mathbf{8 . 1 2}(+/-0.8)$ & $\mathbf{8 . 1}(+/-0.67)$ & 0.96 \\
OBKW (mg/g BWs) & $\mathbf{7 . 5 2}(+/-0.9)$ & $\mathbf{7 . 8 1}(+/-0.96)$ & 0.58 \\
OBKW/CLKW (mg/mg) & $\mathbf{0 . 9 1}(+/-0.08)$ & $\mathbf{0 . 9 7}(+/-0.14)$ & 0.41 \\
\hline
\end{tabular}

Body and kidney weights of both treatment groups. Average (+/- standard deviation) is shown.

$B W$ body weight, $C L K W$ contralateral kidney weight, $O B K W$ obstructed kidney weight 
obstructed kidneys of vehicle treated mice $(P=0.3$; Fig. 1a, b). Also, in the tubulointerstitial compartment of the kidney cortex there was a trend towards reduction of GFP-positive surface area, and GFP signal intensity was slightly reduced upon obstruction (Suppl. Figure 1a-c). Remarkably, rhBMP7 treatment induced only very limited, non-significant increase of glomerular GFP fluorescence and altered neither total GFP positive surface area nor fluorescent signal intensity in GFP positive tubulointerstitial areas (Fig. 1a, b, Suppl. Figure 1a-c respectively).
Comparison between GFP signal detected by direct fluorescence and immunohistochemically (IHC) revealed that IHC detection is more sensitive (Suppl. Figure 2). Using morphometric analysis, percentage positive area of total cortical sections, tubulointerstitial (TI) compartment and glomeruli was analysed in sections where GFP was detected by ImageJ, thus allowing assessment whether canonical BMP signaling increases in cortical areas where normally signalling is absent or below detection limit (Suppl. Figure 3). Average total and TI GFP positive area tended to be lower in
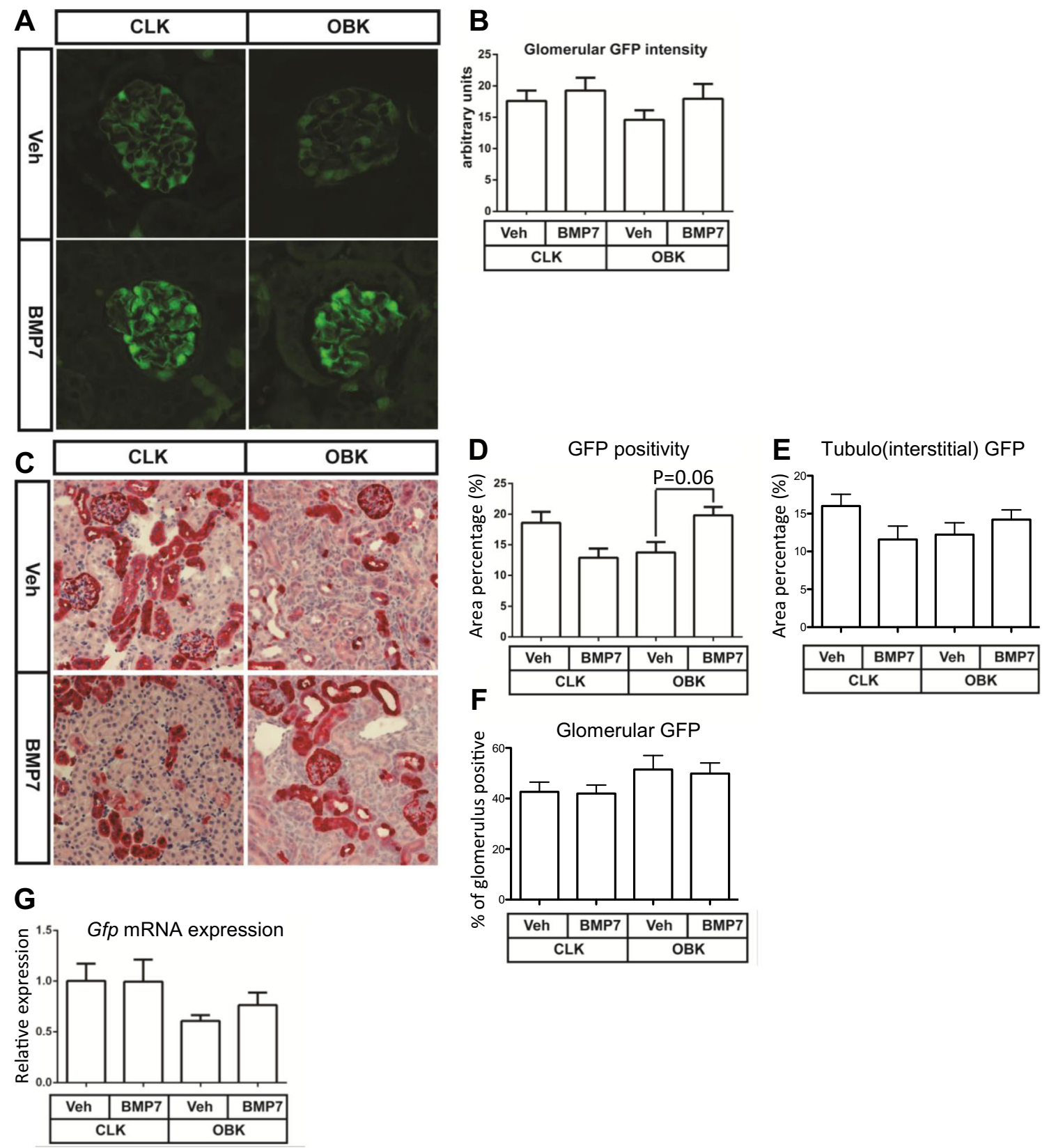

Fig. 1 rhBMP7 treated BRE:Gfp OBKs do not show increased GFP expression. a Representative CLSM micrographs of direct glomerular GFP expression (pseudo colorized). b Quantification of GFP intensity. C. Representative images of GFP immunohistochemistry in CLKs and

OBKs 7 days after UUO of both vehicle and BMP7 groups (200× magnified). d-f. Quantification of total area (B), tubules and interstitium (C) or percentage of the glomeruli (D) positive for GFP. G. Cortical Gfp mRNA expression. Error bars represent SEM 
OBKs than in CLKs, but the observed difference was not significant (Fig. 1d, e). Remarkably, also the apparently small increase of total cortical GFP positive area in rhBMP7 treated OBKs was not statistically significant (Fig. 1d; $p=0.06$ ), and totally lost when tubules and glomeruli were analysed separately (Fig. 1e, f). Analysis of cortical Gfp mRNA expression revealed a significant decrease upon ureteral obstruction, but also here no effect of BMP7 treatment was observed (Fig. 1g).

\section{BMP7 protects against kidney damage and reduces macrophage infiltration 7 days post-UUO}

In PAS stained kidney sections the increase of tubular atrophy and dilatation in OBKs was attenuated in BMP7-treated mice $(p<0.005$; Fig. 2a, b). Lotus tetragonolobus Agglutinin
(LTA) staining revealed that the number of LTA+ proximal tubules was decreased in OBKs compared to CLKs, but less so in BMP7 treated mice (Fig. 2c, d; $p<0.01$ ) Additionally, the OBK/CLK ratio was significantly higher in rhBMP7 treated animals $(p<0.05)$.

Macrophage infiltration in OBKs, as assessed by F4/80 IHC, was markedly reduced in the BMP7 treated group (p $<0.005$; Fig. 2e, f).

\section{Fibrogenesis is reduced in BMP7 treated OBKs}

After 7 days of obstruction, the OBKs of vehicle treated mice showed only a little increase in fibrosis (Suppl. Figure 4). Accumulation of myofibroblasts in OBKS, as assessed by $\alpha$ SMA positive surface area, was decreased by BMP7
Fig. 2 rhBMP7 treatment conserves renal morphology and limits macrophage accumulation 7 days after obstructive nephropathy. a Representative images of PAS stained cortical sections in CLKs and OBKs 7 days after UUO of both vehicle and BMP7 treated groups (200× magnified). b Composite of atrophy and dilatation score quantified on PAS stained slides $\mathbf{c}$ Representative images of LTA stained cortical sections in CLKs and OBKs of both vehicle and BMP7 treated groups (200× magnified). d Quantification of the average number of LTA+ proximal tubules per HPF in CLKs and OBKs of vehicle and rhBMP7 treated kidneys. e Representative images of F4/80 stained cortical sections in CLKs and OBKs of both vehicle and BMP7 treated groups (200× magnified). f Positive area quantification of $\mathrm{F} 4 / 80$ positive macrophages in CLKs and OBKs of vehicle and rhBMP7 treated kidneys. ${ }^{*} p<0.05$, $* * * p<0.005$. Error bar represents SEM. Vehicle $n=6, \operatorname{rhBMP} 7 n=9$
A CLK
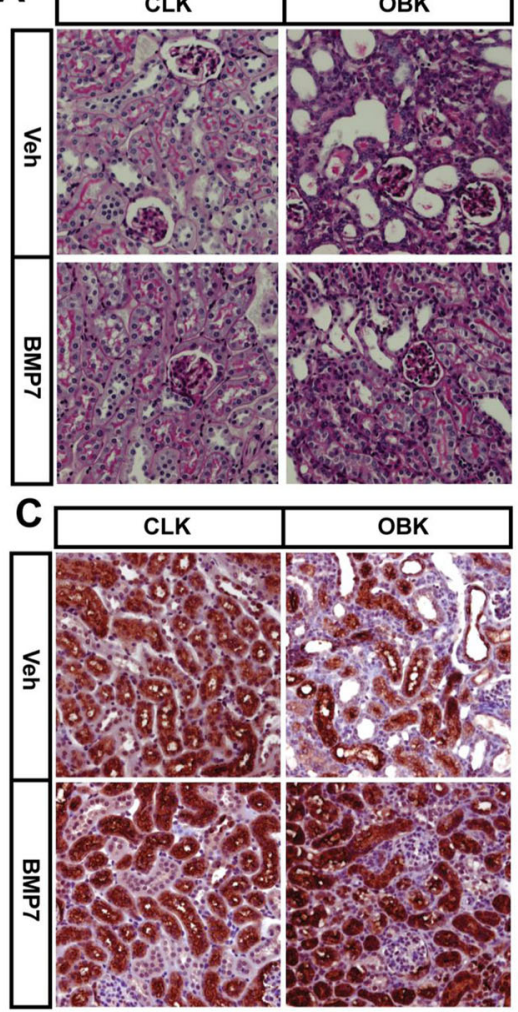

E

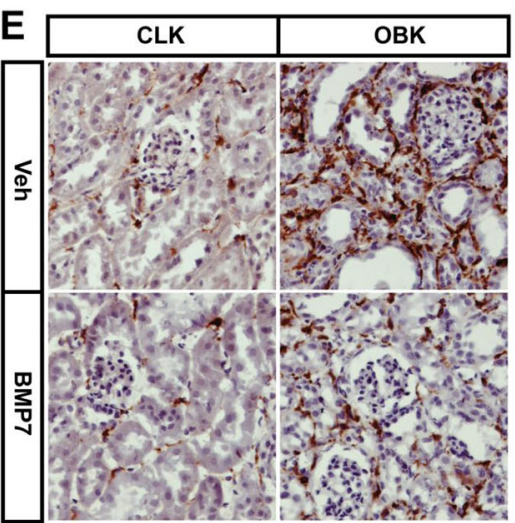

B
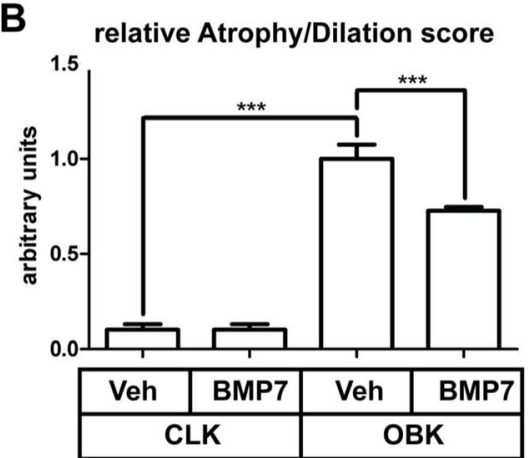

D

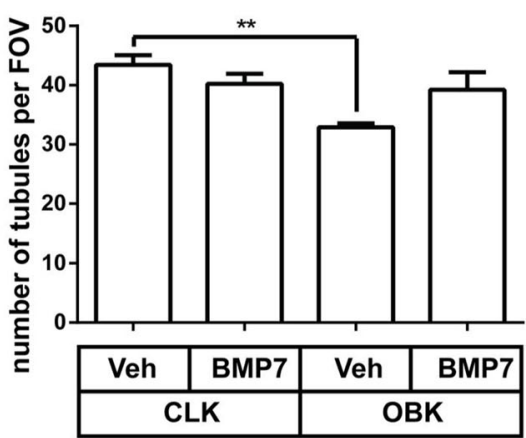

$F$

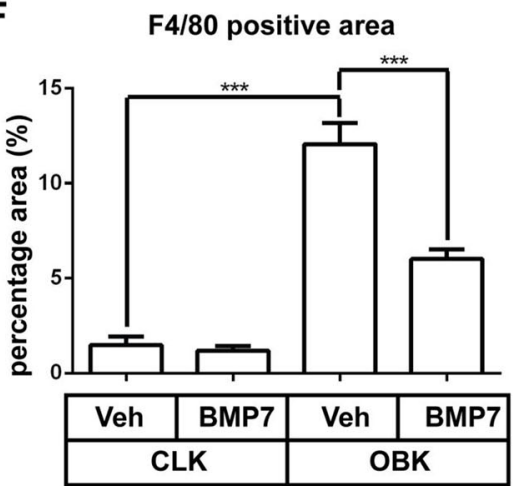


treatment ( $p<0.005$; Fig. 3a, b). Furthermore, the increase of mRNA for $\alpha$-sma and Coll $\alpha 2$ was reduced by BMP7 treatment ( $p<0.01$ and $\mathrm{p}<0.005$ resp.; Fig. $3 \mathrm{c}, \mathrm{d}$ ).

\section{BMP7 treatment reduced CCN2 expression}

\section{but did not alter $\mathrm{Tgf} \beta 1$ expression and transcriptional} activity

Both $\operatorname{Tg} f \beta 1$ and its canonical transcriptional target Pai-1/ SerpinE1 were upregulated in OBKs $(\mathrm{p}<0.005$ and $\mathrm{p}<0.01$ resp.; Fig. 4a, b), which was not affected by BMP7 treatment. However, BMP7 blocked the increase of CCN2 expression in OBKs ( $p<0.05$; Fig. $4 \mathrm{c}, \mathrm{d})$.

\section{In CCN2 hemizygous KO mice, BMP7 treatment} did not further reduce damage, macrophage infiltration, and fibrosis of obstructed kidneys

In good agreement with previous reports, we observed less severe kidney damage, macrophage infiltration, and fibrosis in 7 day OBKs of CCN2 hemizygous KO, than in those of wild type mice. (Suppl. Figure 5a-e). In order to investigate BMP7 induced reno-protection beyond $C C N 2$ reduction, we also treated CCN2+/- UUO mice with rhBMP7. The approximately $50 \%$ reduction of $C C N 2$ expression in $\mathrm{CTGF}+/-$ mice was not further reduced by rhBMP7 treatment (Fig. 5a). In association with this finding, administration of BMP7 to CCN2 hemizygous KO mice also failed to further attenuate renal damage, macrophage infiltration or myofibroblast accumulation (Fig. 5b-d), which emphasizes the importance of $\mathrm{CCN} 2$ reduction in mediating the protective effect of BMP7 treatment in this model of obstructive nephropathy.

\section{Discussion}

The present study confirms that rhBMP7 treatment reduces the severity of kidney damage, macrophage infiltration, and myofibroblast accumulation in a mouse model of obstructive nephropathy (UUO). Seven days after UUO, little to no manifest fibrosis was present (Suppl. Figure 3), which might reflect the relative resistance of C57B16 mice to renal fibrosis in general (Walkin et al. 2013). However, analysis of Coll $\alpha 2$ transcription revealed that de-novo collagen type 1 production was reduced in rhBMP7 treated mice. Remarkably, transcriptional activity downstream of canonical BMP signaling, and
Fig. 3 rhBMP7 treatment reduces myofibroblast accumulation and associated de novo collagen production. a Representative images of $\alpha$ SMA in CLKs and OBKs 7 days after UUO of both vehicle and BMP7 treated groups $(200 \times$ magnified). b Positive area quantification of $\alpha$ SMA positive myofibroblasts in CLKs and OBKs of vehicle and rhBMP7. $\mathbf{c}$ and $\mathbf{d} \alpha$ Sma mRNA (C) and Col1 $\alpha 2$ (D) expression levels in kidney cortex of CLKs and OBKs 7 days after UUO of vehicle and rhBMP7 treated animals. $* \mathrm{p}<0.05, * * p<0.01$, $* * * \mathrm{p}<0.005$. Error bars represent SEM
A

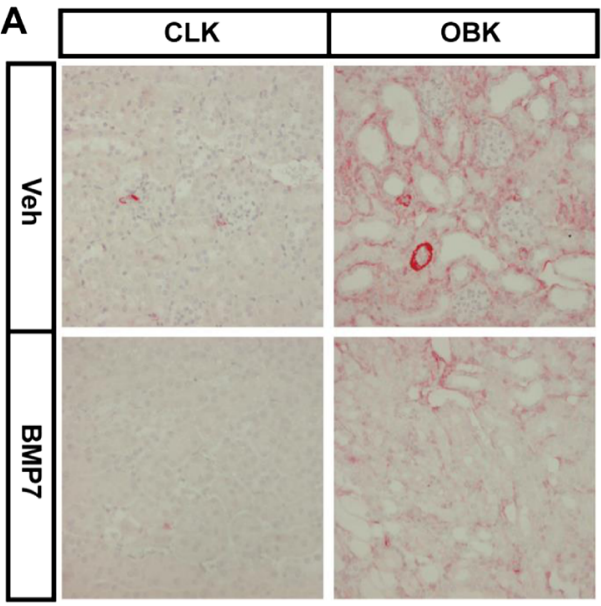

C aSMA mRNA expression

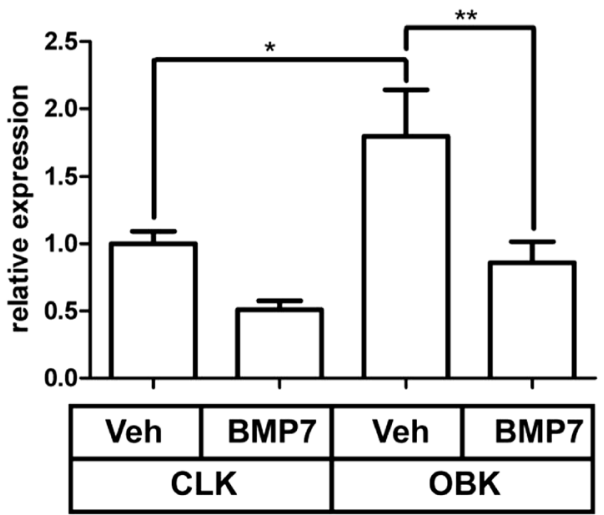

aSMA positive area

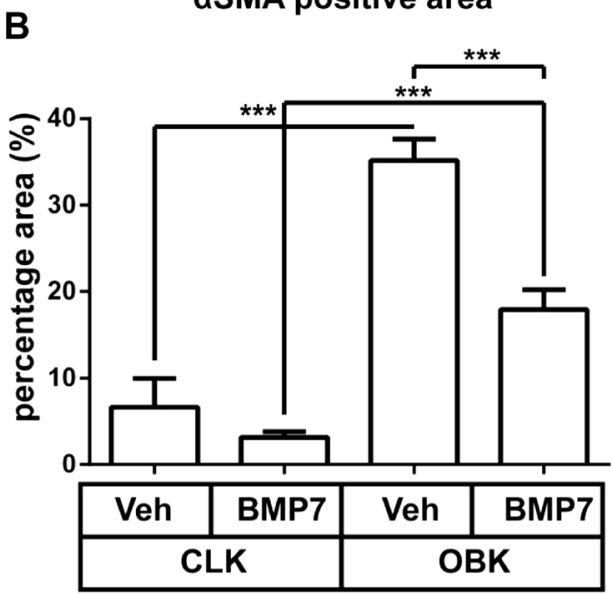

D Col1 $\alpha 2$ mRNA expression

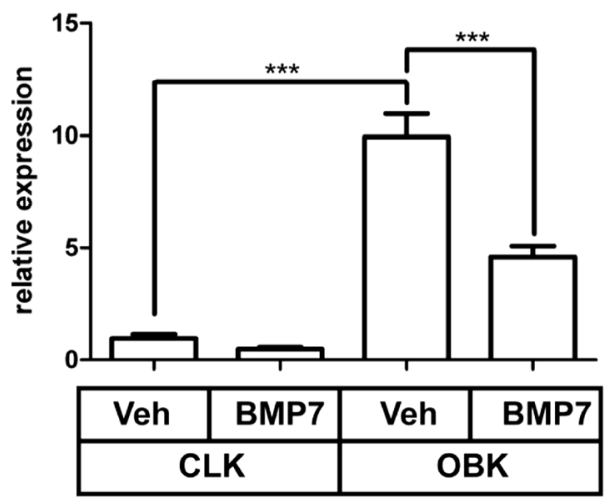


also TGF $\beta$ expression and transcriptional activity, appeared not to be altered by rhBMP7 treatment, while the expression of $\mathrm{CCN} 2$ was significantly reduced in the obstructed kidneys (OBK) of BMP7-treated mice.

In line with previous observations in the BRE-GFP reporter mouse also used here, the GFP signal was slightly reduced in distal tubuli of obstructed kidneys (Fig. 1), but we detected no increase of GFP signal upon rhBMP7-treatment (Manson et al. 2015). Moreover, direct fluorescence of glomeruli and tubulointerstitium showed that established canonical BMP signalling was not increased in intensity nor in terms of positive surface area (Fig. 1a, b, Suppl. Figure 2a-d respectively). Also with more sensitive immunohistochemical detection of GFP protein detection, there was no increase of GFP positive area percentage, indicating that BMP7 did not induce de novo canonical BMP transcriptional activity in previously negative cells (Fig. 1c-f). Unfortunately given its non-stoichiometric nature, IHC signal intensity analysis was not feasible. However, the lack of increase of transcriptional activity downstream of canonical BMP signaling was further underlined by equal GFP mRNA expression levels in renal cortex of rhBMP7 treated and untreated mice (Fig. 1g). It thus appears that the protective effects of rhBMP7 treatment in the 7 days UUO model does not require or involve an increase of canonical
BMP transcriptional activity. This suggests that the beneficial effects of BMP7 treatment mainly involve non-canonical signaling, rather than transcriptional activity downstream of canonical BMP signaling. However, since no data are available on pharmacokinetics of i.p. injected rhBMP7, we cannot fully exclude that rapid elimination or degradation of de novo synthesized GFP protein within the $24 \mathrm{~h}$ window between the last BMP7 dose and sacrifice might have "quenched" the reporter signal.

Previously, we have shown that BMP7 treatment of renal interstitial fibroblasts reduces CCN2 and PAI-1 expression (Nguyen et al. 2006), and Wang et al. demonstrated that BMP7 treatment of mesangial cells reduces CCN2, Fibronectin and Collagen type 4 expression (Wang and Hirschberg 2003). BMP7 also exerts protective effects on TGF- $\beta$ stimulated podocytes (Mitu et al. 2007). As for proximal tubular epithelial cells, Zeisberg et al. showed that BMP7 can inhibit and even reverse TGF $\beta$ driven epithelial to mesenchymal transdifferentiation, although this could not be confirmed by Dudas et al. (Zeisberg et al. 2003; Dudas et al. 2009). In aggregate, it thus appears that rhBMP7 can reduce (TGF $\beta$-induced) $\mathrm{CCN} 2$ expression and associated fibrotic changes in most cell types of the kidney. However, most evidence for this derives from in vitro cultures only, and the
Fig. 4 rhBMP7 treatment reduces $\mathrm{CCN} 2$ expression levels without altering canonical TGF $\beta$ signaling. a-c Cortical mRNA expression levels of $\operatorname{Tgf} \beta 1$ (A), Pai-1 (B) and CCN2 (C) in CLKs and OBKs 7 days after UUO of vehicle and rhBMP7 treated CCN2+/- mice. d Representative images of CCN2 immunohistochemistry in CLKs and OBKs of both vehicle and rhBMP7 treated groups $(200 \times$ magnified). $* \mathrm{p}<0.05, * * \mathrm{p}<0.01$, $* * * \mathrm{p}<0.005$. Error bars represent SEM
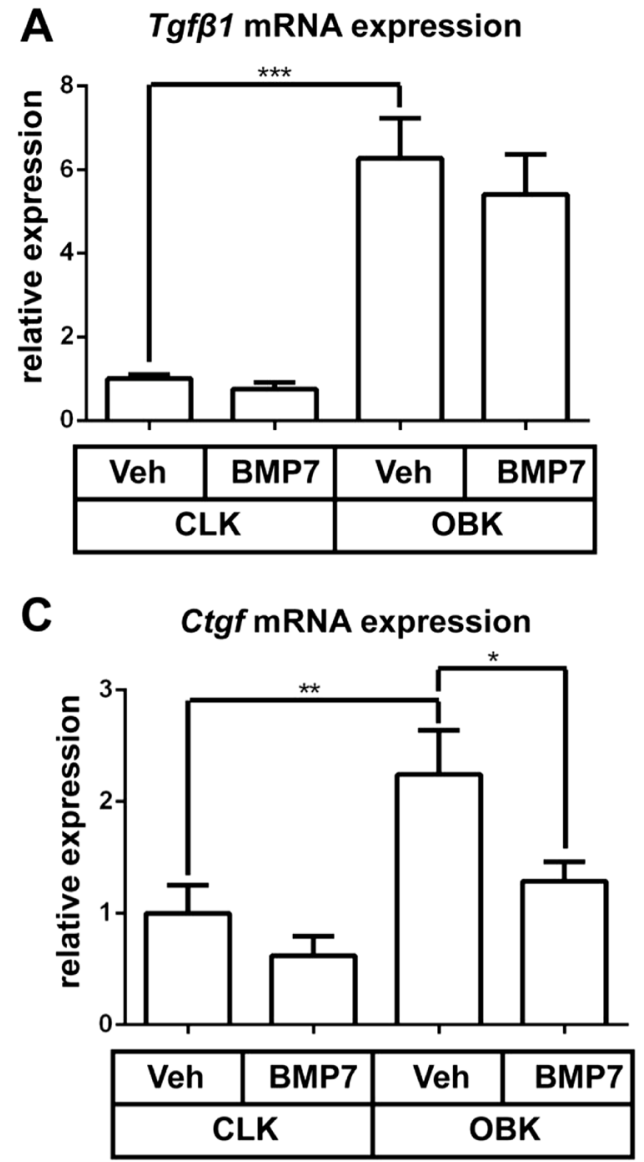
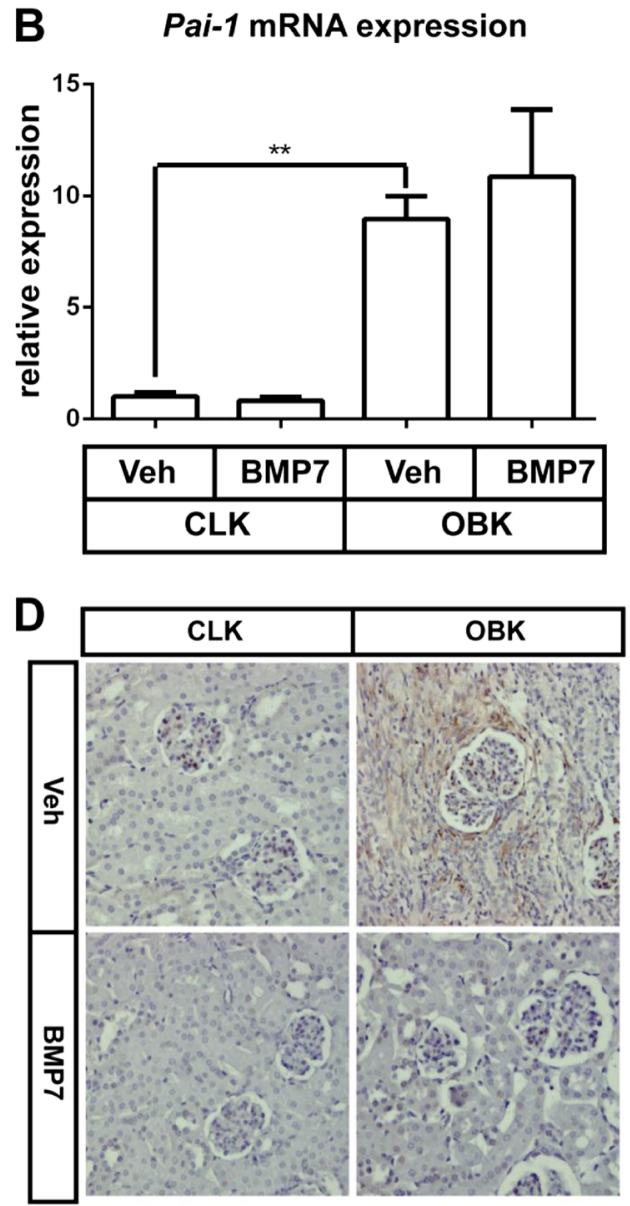
relative contribution of canonical as compared to noncanonical signaling in this has not yet been clearly delineated. The renoprotective effects of rhBMP7 were associated with approximately $50 \%$ reduction of CCN2 expression, whereas expression of $\operatorname{Tg} f \beta 1$ and its prototypic canonical transcriptional target Pai-1 remained unaltered (Fig. 4). Efficacy of BMP7 therapy without altering TGF $\beta$ signaling has been reported previously in a model of diabetic nephropathy (Wang et al. 2006). Of note, hemizygous CCN2 deletion (with $50 \%$ reduced $\mathrm{CCN} 2$ expression), appeared equally effective as rhBMP7 treatment, with similar reduction of morphological damage, macrophage infiltration, and collagen and $\alpha$-SMA expression (Suppl. Figure 5). This is consistent with previous observations that an approximately $50 \%$ reduction of CCN2 expression by genetic deletion or siRNA was sufficient to significantly attenuate models of diabetic nephropathy and obstructive nephropathy (Yokoi et al. 2004; Guha et al. 2007; Nguyen et al. 2008). With respect to possible concerns regarding potential a confounding effect of CCN2suppression prior- and unrelated to the induction of obstructive nephropathy, the reduced CCN2 expression in conventional $\mathrm{CCN} 2+/-$ mice, did not lead to any renal abnormalities during development or adult life, and neither did a $90 \%$ CCN2 reduction sustained for several weeks (Ivkovic et al. 2003; Falke et al. 2012; Falke et al. 2014).

Interestingly, administration of rhBMP7 to heterozygous $\mathrm{CCN} 2$ mice tended to even further reduce $\mathrm{CCN} 2$ expression, but this was not associated with a further decrease in damage and fibrosis. It thus appears that the observed therapeutic effects might relate to a threshold effect of CCN2 reduction rather than on a continuous dose-response relation (Fig. 5).

In summary, in the mouse UUO model of obstructive nephropathy we observed efficacy of rhBMP therapy in the absence of clear evidence for modulation of transcriptional activity downstream of canonical BMP signaling. As a consequence, the BRE-GFP reporter mice failed to reveal the
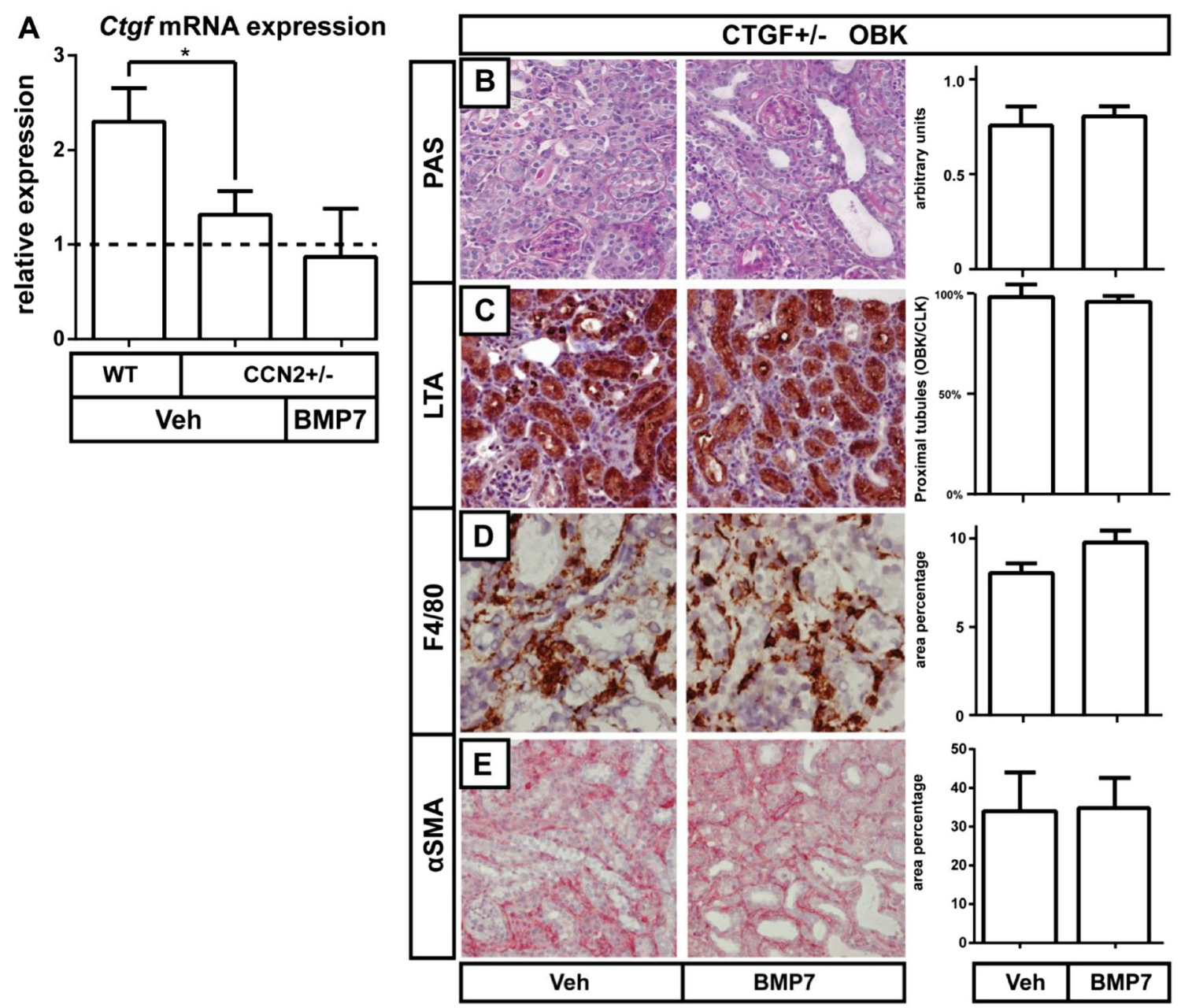

Fig. 5 Treatment with rhBMP7 offers no additional renoprotection in CCN2+/- heterozygous KO mice. a Average cortical mRNA expression levels of CCN2 in CLKs and OBKs 7 days after UUO of vehicle and rhBMP7 treated mice. b-e. Representative micrographs (200× magnified) and corresponding quantification of morphological composite damage score
(B), LTA positive proximal tubules (C), F4/80 positive macrophages (D) and $\alpha$ SMA positive myofibroblasts (E), in OBKs 7 days after UUO of vehicle and rhBMP7 treated $\mathrm{CCN} 2+/-$ mice. $* p<0.05$. Error bars represent SEM 
identity of specific phenotypes and localization of cells responding to exogenous BMP7 therapy. Furthermore, efficacy of BMP7 treatment appeared not to require reduction of TGF $\beta$ expression or transcriptional activity, but was associated with a reduction of CCN2 expression. Hemizygous deletion of CCN2 was able to reduce UUO severity and BMP7 had no additional effect. Together, these data suggest that protection against obstructive nephropathy by exogenous BMP7 treatment relates primarily to non-canonical BMP signalling. Possibly, BMP7 effects are mediated by downregulation of CCN2 expression.

Acknowledgments We acknowledge Stryker medical for kindly providing the rhBMP7 used in this study. Furthermore, we thank Roel Broekhuizen for his valuable technical assistance.

Open Access This article is distributed under the terms of the Creative Commons Attribution 4.0 International License (http:// creativecommons.org/licenses/by/4.0/), which permits unrestricted use, distribution, and reproduction in any medium, provided you give appropriate credit to the original author(s) and the source, provide a link to the Creative Commons license, and indicate if changes were made.

\section{References}

Abreu JG, Ketpura NI, Reversade B, De Robertis EM (2002) Connectivetissue growth factor (CTGF) modulates cell signalling by BMP and TGF- $\beta$. Nat Cell Biol. doi:10.1038/ncb826

Akhurst RJ, Hata A (2012) Targeting the TGF $\beta$ signalling pathway in disease. Nat Rev Drug Discov 11:790-811. doi:10.1038/nrd3810

Dendooven A, van Oostrom O, van der Giezen DM et al (2011) Loss of endogenous bone morphogenetic protein- 6 aggravates renal fibrosis. Am J Pathol 178:1069-1079. doi:10.1016/j.ajpath.2010.12.005

Dube PH, Almanzar MM, Frazier KS et al (2004) Osteogenic protein-1: gene expression and treatment in rat remnant kidney model. Toxicol Pathol 32:384-392

Dudas PL, Argentieri RL, Farrell FX (2009) BMP-7 fails to attenuate TGF-beta1-induced epithelial-to-mesenchymal transition in human proximal tubule epithelial cells. Nephrol Dial Transplant 24:14061416. doi:10.1093/ndt/gfn662

Dudley AT, Lyons KM, Robertson EJ (1995) A requirement for bone morphogenetic protein-7 during development of the mammalian kidney and eye. Genes Dev 9:2795-2807

Falke LL, Dendooven A, Leeuwis JW et al (2012) Hemizygous deletion of $\mathrm{CTGF} / \mathrm{CCN} 2$ does not suffice to prevent fibrosis of the severely injured kidney. Matrix Biol 31:421-431. doi:10.1016 /j.matbio.2012.06.002

Falke LL, Goldschmeding R, Nguyen TQ (2014) A perspective on antiCCN2 therapy for chronic kidney disease. Nephrol Dial Transplant 29(Suppl 1):i30-i37. doi:10.1093/ndt/gft430

Guha M, Xu Z-G, Tung D et al (2007) Specific down-regulation of connective tissue growth factor attenuates progression of nephropathy in mouse models of type 1 and type 2 diabetes. FASEB J 21: 3355-3368. doi:10.1096/fj.06-6713com

Hruska KA, Guo G, Wozniak M et al (2000) Osteogenic protein-1 prevents renal fibrogenesis associated with ureteral obstruction. Am J Physiol Ren Physiol 279:F130-F143
Ito Y, Aten J, Bende RJ et al (1998) Expression of connective tissue growth factor in human renal fibrosis. Kidney Int 53:853-861. doi: $10.1111 / j .1523-1755.1998 .00820 . x$

Ivkovic S, Yoon BS, Popoff SN et al (2003) Connective tissue growth factor coordinates chondrogenesis and angiogenesis during skeletal development. Development 130:2779-2791

Korchynskyi O, Dijke ten P (2002) Identification and functional characterization of distinct critically important bone morphogenetic protein-specific response elements in the Id1 promoter. J Biol Chem 277:4883-4891. doi:10.1074/jbc.M111023200

Leeuwis JW, Nguyen TQ, Chuva de Sousa Lopes SM, et al (2011) Direct visualization of Smad1/5/8-mediated transcriptional activity identifies podocytes and collecting ducts as major targets of BMP signalling in healthy and diseased kidneys. J Pathol 224:121-132. doi:10.1002/path.2844

Manson SR, Song JB, Guo Q et al (2015) Cell type specific changes in BMP-7 expression contribute to the progression of kidney disease in patients with obstructive uropathy. J Urol 193:1860-1869. doi:10.1016/j.juro.2014.10.117

Meng X-M, Tang PM-K, Li J, Lan HY (2015) TGF- $\beta$ /Smad signaling in renal fibrosis. Front Physiol 6:82. doi:10.3389/fphys.2015.00082

Mitu GM, Wang S, Hirschberg R (2007) BMP7 is a podocyte survival factor and rescues podocytes from diabetic injury. Am J Physiol Ren Physiol 293:F1641-F1648. doi:10.1152/ajprenal.00179.2007

Monteiro RM, de Sousa Lopes SMC, Bialecka M et al (2008) Real time monitoring of BMP Smads transcriptional activity during mouse development. Genesis 46:335-346. doi:10.1002/dvg.20402

Morrissey J, Hruska K, Guo G et al (2002) Bone morphogenetic protein-7 improves renal fibrosis and accelerates the return of renal function. $\mathrm{J}$ Am Soc Nephrol 13(Suppl 1):S14-S21

Muñoz-Félix JM, González-Núñez M, Martínez-Salgado C, LópezNovoa JM (2015) TGF- $\beta$ /BMP proteins as therapeutic targets in renal fibrosis. Where have we arrived after 25 years of trials and tribulations? Pharmacol Ther 156:44-58. doi:10.1016/j. pharmthera.2015.10.003

Nguyen TQ, Chon H, van Nieuwenhoven FA et al (2006) Myofibroblast progenitor cells are increased in number in patients with type 1 diabetes and express less bone morphogenetic protein 6: a novel clue to adverse tissue remodelling? Diabetologia 49:1039-1048. doi:10.1007/s00125-006-0172-0

Nguyen TQ, Roestenberg P, van Nieuwenhoven FA et al (2008) CTGF inhibits BMP-7 signaling in diabetic nephropathy. J Am Soc Nephrol 19:2098-2107. doi:10.1681/ASN.2007111261

Ren Y, Du C, Yan L et al (2015) CTGF siRNA ameliorates tubular cell apoptosis and tubulointerstitial fibrosis in obstructed mouse kidneys in a Sirt1-independent manner. Drug Des Devel Ther 9:4155-4171. doi:10.2147/DDDT.S86748

Sugimoto H, Grahovac G, Zeisberg M, Kalluri R (2007) Renal fibrosis and glomerulosclerosis in a new mouse model of diabetic nephropathy and its regression by bone morphogenic protein-7 and advanced glycation end product inhibitors. Diabetes 56:1825-1833. doi: $10.2337 / \mathrm{db} 06-1226$

Vukicevic S, Basic V, Rogic D et al (1998) Osteogenic protein-1 (bone morphogenetic protein-7) reduces severity of injury after ischemic acute renal failure in rat. J Clin Invest 102:202-214. doi:10.1172 /JCI2237

Walkin L, Herrick SE, Summers A et al (2013) The role of mouse strain differences in the susceptibility to fibrosis: a systematic review. Fibrogenesis Tissue Repair 6:18. doi:10.1186/1755-1536-6-18

Wang S, Hirschberg R (2003) BMP7 antagonizes TGF-beta -dependent fibrogenesis in mesangial cells. Am J Physiol Ren Physiol 284: F1006-F1013. doi:10.1152/ajprenal.00382.2002

Wang S, Chen Q, Simon TC et al (2003) Bone morphogenic protein-7 (BMP-7), a novel therapy for diabetic nephropathy. Kidney Int 63: 2037-2049. doi:10.1046/j.1523-1755.2003.00035.x 
Wang S, de Caestecker M, Kopp J et al (2006) Renal bone morphogenetic protein-7 protects against diabetic nephropathy. J Am Soc Nephrol 17:2504-2512. doi:10.1681/ASN.2006030278

Yokoi H, Mukoyama M, Nagae T et al (2004) Reduction in connective tissue growth factor by antisense treatment ameliorates renal tubulointerstitial fibrosis. J Am Soc Nephrol 15:1430-1440
Zeisberg M, Hanai J-I, Sugimoto H et al (2003) BMP-7 counteracts TGF-betal-induced epithelial-to-mesenchymal transition and reverses chronic renal injury. Nat Med 9:964-968. doi: $10.1038 / \mathrm{nm} 888$ 